\title{
Avaliação in vitro da eficácia da remoção da guta-percha no retratamento endodôntico por meio de microscopia operatória
}

\author{
In vitro evaluation of the efficacy of gutta-percha removal in endodontic retreatment \\ by operative microscopy \\ Evaluación in vitro de la eficacia de la remoción del guta-percha en el retratamiento \\ endodóncico a través de microscopia operativa \\ Josimeire Alves Pereira BARBOSA ${ }^{1}$ \\ Melissa Ayumi TATEYAMA ${ }^{2}$ \\ Carlos Alberto Herrero de MORAIS ${ }^{3}$ \\ Alfredo Franco QUEIROZ \\ Nair Narumi Orita PAVAN ${ }^{3}$ \\ Marcos Sérgio ENDO ${ }^{3}$ \\ ${ }^{I}$ Graduada em Odontologia - Departamento de Odontologia (DOD), Centro de Ciências da Saúde (CCS), \\ Universidade Estadual de Maringá (UEM) 87083-170 Maringá-PR, Brasil \\ ${ }^{2}$ Graduanda de Odontologia - Departamento de Odontologia (DOD), Centro de Ciências da Saúde (CCS), \\ Universidade Estadual de Maringá (UEM) 87083-170 Maringá -PR, Brasil \\ ${ }_{3}^{3}$ Professor(a) Adjunto(a) em Endodontia - Departamento de Odontologia (DOD), Centro de Ciências da Saúde (CCS), \\ Universidade Estadual de Maringá (UEM) 87083-170 Maringá -PR, Brasil \\ ${ }^{4}$ Professor Assistente em Endodontia - Departamento de Odontologia (DOD), Centro de Ciências da Saúde (CCS), \\ Universidade Estadual de Maringá (UEM) 87083-170 Maringá-PR, Brasil
}

\section{Resumo}

Retratamento endodôntico é uma tentativa de restabelecimento da saúde dos tecidos periapicais devido um tratamento inadequado ou uma reinfecção dos canais obturados que foram acometidos por uma infiltração coronária ou apical. A remoção completa do material obturador é necessária para obter acesso ao forame, facilitando assim a limpeza e modelagem do sistema de canais radiculares. O objetivo deste estudo foi comparar in vitro a remoção da guta-percha com ou sem uso do solvente. Vinte e oito pré-molares unirradiculares foram instrumentados com sistema Reciproc e obturados por meio da técnica de condensação lateral. A remoção da guta-percha foi realizada de duas formas distintas, sendo as amostras divididas aleatoriamente em dois grupos: com $(n=14)$ e sem solvente $(n=14)$. Após o seccionamento dos espécimes, realizou-se a captura das imagens das raízes por meio de uma câmera fotográfica acoplada ao microscópio operatório. A mensuração da área de guta-percha remanescente foi realizada pelo programa AUTOCAD. O remanescente de guta-percha entre os grupos foi comparado por meio do teste Mann-Whitney $(\mathrm{p}<0,05)$. Após aplicação da técnica de desobturação, verificou-se que todas as amostras mostraram uma redução estatisticamente significante. Quando comparados os terços cervical (TC), médio (TM) e apical (TA) do canal radicular no terço apical foi verificado maior quantidade de remanescente de guta-percha em ambos os grupos estudados. Não houve diferença estatística significativa sobre o remanescente de guta-percha ao comparar os grupos com e sem solvente ( $\mathrm{p}=0,546)$. Concluiu-se que o uso do solvente não melhorou a remoção de remanescente de guta-percha das paredes do canal radicular.

Descritores: Endodontia; Guta-Percha; Retratamento; Dente.

\section{Abstract}

Endodontic retreatment is an attempt to reestablish the health of the periapical tissues due to inadequate treatment or reinfection of the filled canals that have been affected by a coronary or apical infiltration. The complete removal of the guta-percha is necessary to gain access to the foramen, facilitating the cleaning and modeling of the canal system. The objective of this study was to compare in vitro the removal of guttapercha with or without solvent. Twenty-eight unirradicular premolars were instrumented with Reciproc system and obturated using the lateral condensation technique. The gutta-percha was removed in two different ways, with the samples randomly divided into two groups: with $(n=14)$ and without solvent $(n=14)$. After the sectioning of the specimens, the images of the roots were captured by means of a photographic camera attached to the operative microscope. The measurement of the remaining gutta-percha area was performed by the AUTOCAD program. The remnant of gutta-percha between the groups was compared using the Mann-Whitney test (p <0.05). After application of the guta-percha removal technique, it was found that all the samples showed a statistically significant reduction. When comparing the cervical (TC), middle (TM) and apical (TA) thirds of the root canal, a greater amount of gutta-percha remnant was observed in both groups. There was no significant statistical difference on the remnant of gutta-percha when comparing the groups with and without solvent $(\mathrm{p}=0.546)$. It was concluded that the use of the solvent did not improve the removal of gutta-percha remnant from the root canal walls.

Descriptors: Endodontics; Gutta-Percha; Retreatment; Tooth.

\section{Resumen}

Retratamiento endodóncico es una tentativa de restablecimiento de la salud de los tejidos periapicales debido a un tratamiento inadecuado o una reinfección de los canales obturados que fueron acometidos por una infiltración coronaria o apical. La remoción completa del material obturador es necesaria para obtener acceso al foramen, facilitando así la limpieza y modelado del sistema de canales radiculares. El objetivo de este estudio fue comparar in vitro la remoción de la gutapercha con o sin uso del diluyente. Veintiocho premolares unirradiculares fueron instrumentados con sistema Reciproc y obturados através de la técnica de condensación lateral. La remoción de la gutapercha fue realizada de dos formas distintas, siendo las muestras divididas aleatoriamente en dos grupos: con $(n=14)$ y sin diluyente ( $n=14)$. Tras el seccionamiento de los especímenes, se realizó la captura de las imágenes de las raíces por medio de una cámara fotográfica aparejada al microscopio operatorio. La medición del área de gutapercha remanente fue realizada por el programa AUTOCAD. El remanente de gutapercha entre los grupos fue comparado a través del test Mann-Whitney $(\mathrm{p}<0,05)$. Tras aplicación de la técnica de desobturación, se comprobó que todas las muestras presentaron una reducción estadísticamente significante. Cuando comparados los tercios cervicales (TC), medio (TM) y apical (TA) del canal radicular en el tercio apical fue verificado mayor cantidad de remanente de gutapercha en ambos grupos estudiados. No hubo diferencia estadística significativa sobre el remanente de gutapercha al comparar los grupos con y sin diluyente $(\mathrm{p}=0,546)$. Se concluyó que el uso del diluyente no mejoró la remoción del remanente de gutapercha de las paredes del canal radicular. Descriptores: Endodoncia; Gutapercha; Retratamiento; Diente.

\section{INTRODUÇÃO}

Retratamento endodôntico é uma tentativa de restabelecimento da saúde dos tecidos periapicais devido um tratamento inadequado ou uma reinfecção dos canais obturados que foram acometidos por uma infiltração coronária ou apical ${ }^{1}$. Quando possível, em casos em que o primeiro tratamento falhou, o 
retratamento endodôntico não cirúrgico é indicado ${ }^{2}$, sendo necessário remover completamente o material obturador para obter acesso ao forame, facilitando assim a limpeza e modelagem do sistema de canais radiculares e melhorando a desinfecção do canal radicular para posterior obturação ${ }^{3}$. A remoção da guta-percha e do cimento é um fator importante no retratamento, pois nesta massa obturadora permanecerá tecido necrosado e micro-organismos que poderão ser os responsáveis pela inflamação periapical ${ }^{4}$.

A remoção do material obturador pode ser realizada por diversas formas, entre elas: limas manuais, instrumentos aquecidos, pontas ultrassônicas ou limas rotatórias/reciprocantes ${ }^{5-11}$. Os solventes são aplicados durante o retratamento endodôntico com objetivo de reduzir a resistência da guta-percha no interior do canal radicular $^{12}$, facilitando a sua remoção ${ }^{13}$, entre os mais utilizados estão o óleo de laranja, eucaliptol, xilol e clorofórmio $^{14-16}$. O xilol foi considerado citotóxico, e o clorofórmio como carcinogênico ${ }^{17,18}$. Dessa forma recomenda-se utilizar um solvente que apresente uma maior biocompatibilidade como o óleo de laranja ${ }^{19-21}$ reduzindo as injúrias aos tecidos periapicais e que ofereça uma adequada remoção do material obturador.

Diante do exposto, o objetivo deste trabalho foi avaliar in vitro, por meio da microscopia operatória, a eficácia da remoção da guta-percha das paredes do canal radicular utilizando limas manuais com ou sem solvente.

\section{MATERIAL E MÉTODO}

Esse trabalho foi aprovado pelo Comitê de Ética em Pesquisa envolvendo Seres Humanos da Universidade Estadual de Maringá (CAAE: 53358216.1.0000.0104).

\section{- Seleção e preparo dos espécimes}

Foram selecionados 28 dentes unirradiculares com canal único, reto $\left(<5^{\circ}\right)$ e raiz completamente formada. Os dentes com tratamento endodôntico prévio, calcificações, reabsorção interna ou externa e outras alterações anatomopatológicas foram excluídos do estudo. Os espécimes foram limpos em cuba ultrassônica e armazenados em solução de timol a $0,1 \%$. Radiografias periapicais pré-operatórias vestíbulo-lingual e mésio-distal foram realizadas para confirmar a presença de um único canal (Figuras 1 e 2). Os espécimes foram seccionados transversalmente (Figuras 3 e 4) utilizando-se disco diamantado dupla face (KG Sorensen, Cotia-SP, Brasil) e padronizados em comprimento radicular de $16 \mathrm{~mm}$ (Figuras 5 e 6). O canal foi irrigado com hipoclorito de sódio 2,5\% ( $\mathrm{NaOCl}$ ), a exploração dos canais e a patência foraminal realizadas com lima do tipo K\#10 (Dentsply Maillefer, Ballaigues, Switzerland).

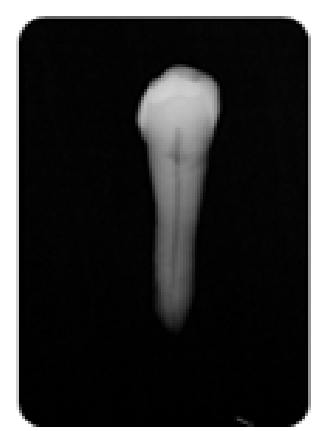

Figura 1: Radiografia periapical vestíbulo-lingual.

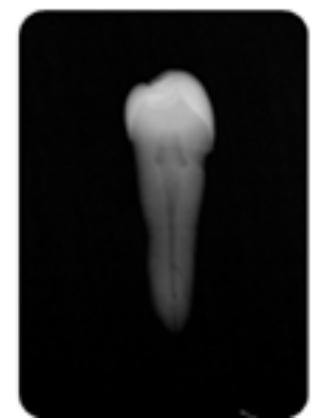

Figura 2: Radiografia periapical mésio-distal.

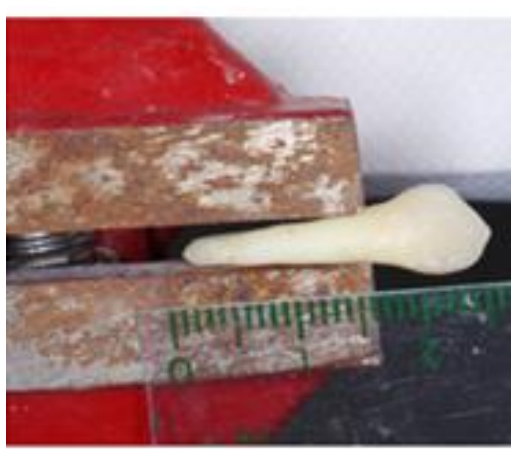

Figura 3: Padronização do espécime.

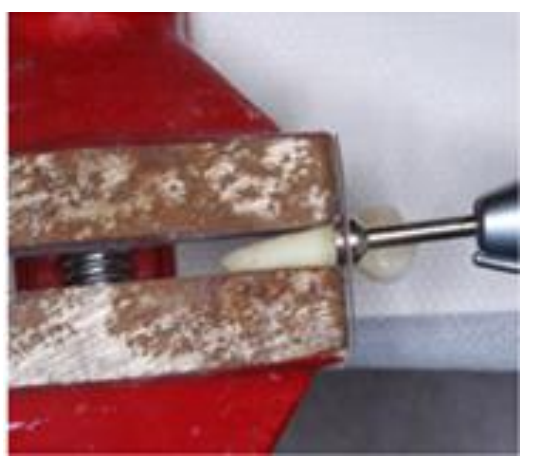

Figura 4: Seccionamento do espécime.

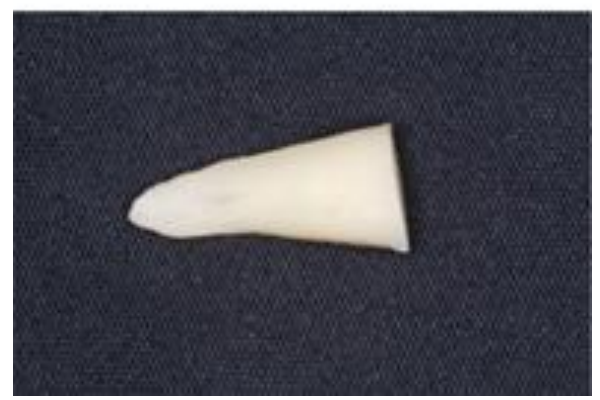

Figura 5: Espécime padronizado após o seccionamento. 


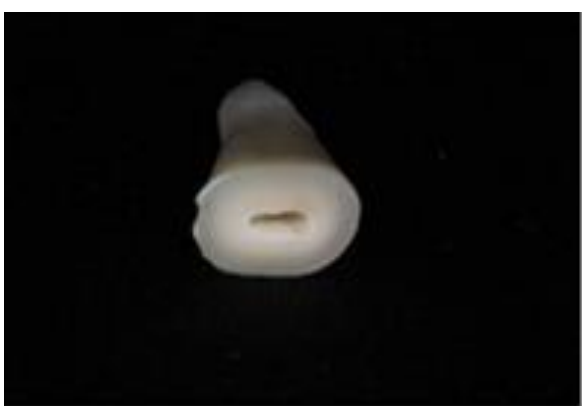

Figura 6: Espécime padronizado após o seccionamento.

\section{- Instrumentação do canal radicular}

A odontometria foi determinada com uma lima do tipo K \#10 (Dentsply Maillefer, Ballaigues, Switzerland) introduzida passivamente no canal até sua ponta ser visualizada no forame apical. $O$ comprimento real de trabalho foi obtido, subtraindo 1 $\mathrm{mm}$ da medida do instrumento. $\mathrm{O}$ canal foi preparado utilizando a técnica crown-down. Os terços cervical e médio foram pré-alargados com a broca Laaxxes 20.06 (Dentsply Maillefer, Ballaigues, Switzerland), e o canal radicular foi instrumentado com as limas Reciproc, R25, R40 e R50 (VDW, Munich, Germany), de acordo com as recomendações do fabricante. A irrigação foi realizada com $25 \mathrm{~mL}$ de solução de $\mathrm{NaOCl} 2,5 \%$ por amostra e aplicado com uma seringa descartável e agulha Navitip 30 ga (Ultradent Products Inc., South Jordan, UT, EUA). A aspiração se deu por meio de uma cânula metálica. Após o preparo foi aplicado $5 \mathrm{~mL}$ de EDTA a $17 \%$ (Biodinâmica, Ibiporã, PR, Brasil) permanecendo por 3 minutos sob agitação com easyclean e uma irrigação final com $5 \mathrm{~mL}$ de $\mathrm{NaOCl}$ 2,5\%. Realizouse a secagem do canal radicular com auxílio de cone de papel absorvente.

\section{- Obturação do canal radicular}

Os canais foram obturados utilizando gutapercha e cimento a base de óxido de zinco e eugenol (Endofill, Dentsply, Petrópolis, RJ, Brasil) por meio da técnica de condensação lateral (Figura 7). A superfície cervical dos espécimes foi limpa com bolinha de algodão embebida em álcool $95^{\circ} \mathrm{GL}$. Cada espécime foi radiografado para verificar o preenchimento do canal radicular. A embocadura do canal foi restaurada com Villevie (Villevie, Joinville, $\mathrm{SC}$, Brasil), e as amostras foram armazenadas sob $100 \%$ de umidade a $37^{\circ} \mathrm{C}$ durante 15 dias.

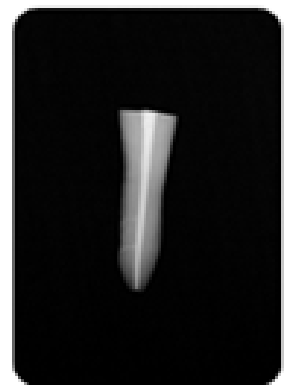

Figura 7. Radiografia periapical para verificação da obturação do canal radicular.
- Divisão dos grupos experimentais e remoção do material obturador

Os 28 espécimes foram divididos aleatoriamente em 2 grupos experimentais contendo 14 dentes cada. Realizou-se a remoção do selamento provisório com broca diamantada esférica \#1012 (KG Sorensen, Cotia, SP, Brasil), acionada em alta rotação e irrigação com $\mathrm{NaOCl} 2,5 \%$. Para ambos os grupos inicialmente foi utilizada broca de Largo \#2 nos primeiros $4 \mathrm{~mm}$ dos canais radiculares para remoção da guta-percha.

I) Grupo com solvente (CS): o canal radicular foi desobturado em toda sua extensão com limas manuais do tipo $\mathrm{K}$ associadas a $0,10 \mathrm{~mL}$ de óleo de laranja (Citrol, Biodinâmica, Ibiporã, PR, Brasil) e irrigado com $25 \mathrm{~mL}$ de $\mathrm{NaOCl} 2,5 \%$.

II) Grupo sem solvente (SS): o canal radicular foi desobturado em toda sua extensão somente com limas manuais do tipo K. A irrigação foi realizada com $25 \mathrm{~mL}$ de solução de $\mathrm{NaOCl}$ 2,5\%.

Realizou-se a reinstrumentação com a lima memória e outra lima com um calibre superior a esta. Para não ocorrer variação inter-operador, o experimento foi realizado por um único operador.

- Avaliação da eficácia da remoção da guta-percha e análise estatística

Os dentes foram seccionados no sentido longitudinal na face vestibular e lingual utilizando disco diamantado (Microdont, São Paulo, Brasil) e divididos em duas metades (mesial e distal). Todas as amostras foram codificadas e então fotografadas, sob uma magnificação de 10x. As imagens foram capturadas por meio de uma câmera fotográfica digital (Nikon, Tóquio, Japão) acoplada ao microscópio operatório (DF Vasconcellos, São Paulo, Brasil).

As imagens capturadas foram transferidas para o computador e a área de guta-percha remanescente foi demarcada no programa AUTOCAD (2015) (Figura 8).

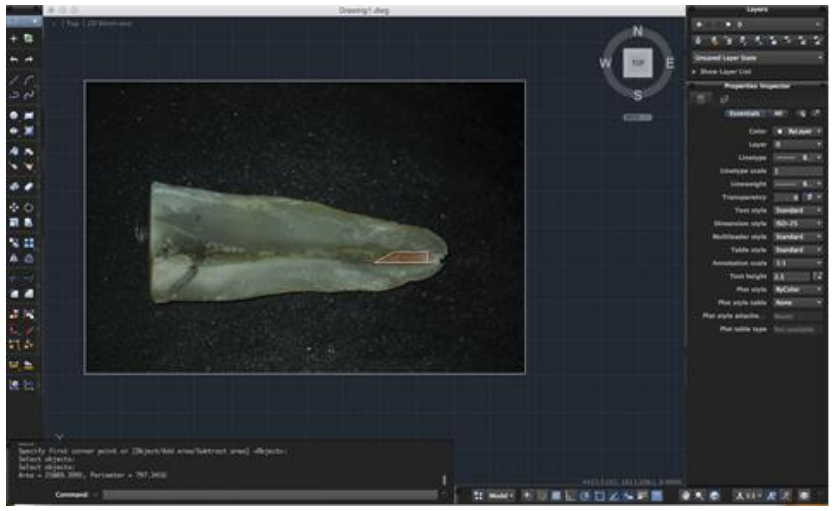

Figura 8: Imagens capturadas para obtenção da área de guta-percha remanescente no programa AUTOCAD (2015).

A distribuição normal dos dados foi verificada pelo teste de Shapiro-Wilk $(\mathrm{p}<0,05)$ e os valores obtidos da guta-percha remanescente foram 
comparados estatisticamente (teste não paramétrico de Mann-Whitney, $\mathrm{p}<0,05$ ), utilizando o programa SIGMAPLOT $12^{\circledR}$. Além disso, também aplicou-se o teste-t pareado $(\mathrm{p}<0,05)$ para comparar a redução da guta-percha antes e após a intervenção do retratamento endodôntico.

\section{RESULTADOS}

Após aplicação da técnica de desobturação, verificou-se que todas as amostras mostraram uma redução estatisticamente significante $(\mathrm{p}<0,001)$. Ao comparar a área total inicial em relação ao remanescente de guta-percha após o procedimento clínico, observou-se uma redução no grupo CS de $96,3 \%$ e no grupo SS $97,5 \%$.

Nas amostras do grupo CS, sua mediana em relação ao valor final do remanescente de guta-percha representou a 0,938 , enquanto que no grupo SS este valor foi de 0,682 . Apesar disso, não houve diferença estatística significante entre os grupos CS e SS $(\mathrm{p}=0,546)$.

Quando comparados os terços cervical (TC), médio (TM) e apical (TA) do canal radicular; os valores médios dos remanescentes de guta percha no grupo CS foram 0,02 (TC), 0,2 (TM) e 1,4 (TA), e no grupo SS corresponderam a 0,03 (TC), 0 (TM) e 1,08 (TA).

\section{DISCUSSÃO}

No retratamento endodôntico há necessidade da remoção do material obturador prévio, objetivando uma melhor limpeza das paredes do canal radicular e remoção de debris teciduais em ramificações anatômicas assim como em túbulos dentinários ${ }^{1,22^{3}}$.

Nossos resultados estão de acordo com a literatura, em que se verifica grande dificuldade da remoção completa da guta-percha nas paredes do canal radicular ${ }^{23}$, e que a respeito do método utilizado para desobturação, nenhum método é capaz de remover totalmente o material obturador ${ }^{5,12,24}$. Quando avaliados os terços cervical, médio e apical; no terço apical foi verificado maior quantidade de remanescente de guta-percha em ambos os grupos estudados corroborando com os resultados dos trabalhos de Masiero et al. ${ }^{24}$, Ezzie et al. $^{25} \mathrm{e}$ Mollo et al. ${ }^{26}$.

Estudos anteriores sobre a remoção da gutapercha dos canais radiculares foram realizados visando avaliar e quantificar quão eficiente era a desobturação por meio de diferentes métodos e técnicas como a avaliação radiográfica ${ }^{24,27}$ ou seccionamento longitudinal das raízes e mensuração do material remanescente ${ }^{28}$. Além desses, outros métodos foram utilizados para analisar $\mathrm{o}$ remanescente do material obturador, sendo por meio da tomografia computadorizada ${ }^{29}$, microtomografia computadorizada $^{30}$, imagens fotográficas de dentes $\operatorname{seccionados}^{31}$ e microscopia eletrônica de varredura $^{25,32}$. Optou-se em utilizar a microscopia operatória por poder ser utilizado durante a intervenção clínica do retratamento endodôntico e ser uma ferramenta que auxilia na magnificação e iluminação do campo de trabalho ${ }^{33}$. Para Schirrmeister et al. ${ }^{34}$ a utilização do microscópio operatório evidencia a presença de guta-percha que não é visível radiograficamente, porém a metodologia usada durante o seccionamento pode ser considerada destrutiva removendo material durante sua preparação, o que não ocorre na avaliação com a microtomografia computadorizada.

A dificuldade na remoção da guta-percha no canal radicular é frequentemente ditada pelas características anatômicas e pela qualidade da obturação. Nesses casos, a associação a um solvente, pode ser um coadjuvante extremamente válido, pois nessas situações observa-se dificuldade no acesso aos canais radiculares ${ }^{25}$. Entretanto, no presente estudo não foi detectada diferença estatisticamente significante na remoção da guta-percha quando comparado os grupos SS e CS.

Devido o estudo de Marques et al. $^{35}$ realizou-se o alargamento com outra lima de calibre superior ao instrumento memória para melhorar a remoção do material obturador. Outros recursos como a irrigação ultrassônica passiva do $\mathrm{NaOCl}$ e EDTA tem sido utilizados para auxiliar na limpeza da remoção de tecidos orgânicos remanescentes, debris e micro-organismos presentes nas paredes dos canais radiculares e áreas de difícil acesso ${ }^{36}$.

CONCLUSÃO

Concluiu-se que a técnica de retratamento preconizada reduziu significativamente a quantidade de guta-percha das paredes do canal radicular, e o terço apical foi a região mais difícil de se remover o material obturador. Além disso, o uso do solvente não melhorou a remoção de remanescente de gutapercha das paredes do canal radicular.

\section{REFERÊNCIAS}

1. Stabholz A, Friedman S. Endodontic retreatment - case selection and technique. Part 2: Treatment planning for retreatment. J Endod. 1988; 14(12):607-14.

2. Barrieshi-Nusair KM. Gutta-percha retreatment: effectiveness of Nickel-Titanium rotary instruments versus stainless steel hand files. J Endod. 2002; 28(6):454-56.

3. Ruddle CJ. Nonsurgical retreatment. J Endod. 2004; 30(12):827-45.

4. Siqueira JF, Roças IN. Polymerase chain reaction-based analysis of microorganisms associated with failed endodontic treatment. Oral Surg Oral Med Oral Pathol Oral Radiol Endod. 2004; 97(1):85-94.

5. Wilcox LR, Krell KV, Madison S, Rittman B. 
Endodontic retreatment: Evaluation of guttapercha and sealer removal and canal reinstrumentation. J Endod. 1987; 13(9):453-57.

6. Wilcox LR. Endodontic retreatment: ultrasonics and chloroform as the final step in reinstrumentation. J Endod. 1989; 15(3):125-28.

7. Friedman S, Stabholz A, Tamse A. Endodontic retreatment-case selection and technique. 3. Retreatment techniques. J Endod. 1990; 16(11):543-49.

8. Zakariasen KL, Boran T, Macdonald R. The emerging role for lasers in endodontics and other areas of dentistry. Alpha Omegan. 1990; 83(4):65-7.

9. Teplitsky EP, Rayner D, Chin I, Markowsky R. Gutta-percha removal utilizing GPX instrumentation. J Can Dent Assoc. 1992; 58(1):53-8.

10. Hulsmann M, Stotz S. Efficacy, cleaning ability and safety of different devices for gutta-percha removal in root canal retreatment. Int Endod J. 1997;30(4):227-33.

11. Fruchi LC, Ordinola-Zapata R, Cavenago BC, Hungaro MA, Bueno CE, De Martin AS. Efficacy of reciprocating instruments for removing filling material in curved canals obturated with a single cone technique: a microcomputed tomographic analysis. J Endod. 2014; 40(7):1000-4.

12. Bodrumlu E, Er O, Kayaoglu, G. Solubility of root canal sealers with different organic solvents. Oral Surg Oral Med Oral Pathol Oral Radiol Endod. 2008;106(3):67-9.

13. Tasdemir T, Er K, Yildirim T, Celik D. Efficacy of three rotary NiTi instruments in removing gutta-percha from root canals. Int Endod J. 2008; 41(3):191-96.

14. Hunter KR, Doblecki W, Pelleu Jr GB. Halothane and eucalyptol as alternatives to chloroform for softening gutta-percha. J Endod. 1991; 17(7):310-11.

15. Kaplowitz GJ. Evaluation of the ability of essential oils to dissolve gutta-percha. J Endod. 1991; 17(9):448-9.

16. Ladley RW, Campbell AD, Hicks ML, Li SH. Effectiveness of halothane used with ultrasonics or hand instrumentation to remove gutta-percha from the root canal. J Endod. 1991; 17(5):221-4.

17. Kaplowitz GJ. Evaluation of gutta-percha solvents. J Endod.1990; 16(11):539-40.

18. Wourms DJ, Campbell AD, Hicks ML, Pelleu GB Jr, Alternative solventes to chloroform for gutta-percha removal. J Endod. 1990; 16(5):224-26.

19. Hansen MG. Relative efficiency of solvents used in endodontics. J Endod. 1998; 24(1):38-40.

20. Ribeiro D, Matsumoto MA, Marques ME,
Salvadori DM. Biocompatibility of gutta-percha solvents using in vitro mammalian test-system. Oral Surg Oral Med Oral Pathol Oral Radiol Endod. 2007; 103(5):106-9.

21. Mushtaq M, Masoodi A, Farooq R, Yaqoob Khan F. The dissolving ability of different organic solvents on three different root canal sealers: In vitro study. Iran Endod J. 2012; 7(4):198-202.

22. Mandel E, Friedman S. Endodontic retreatment: a rational approach to root canal reinstrumentation. J Endod.1992; 18(11):565-69.

23. Rodig T, Reicherts P.; Konietschke F, Dullin C, Hahn W, Hulsmann M. Efficacy of reciprocating and rotary NiTi instruments for retreatment of curved root canals assessed by micro-CT. Int Endod J. 2014; 47(10):942-8.

24. Masiero AV, Barletta FB. Effectiveness of different techniques for removing gutta-percha during retreatment. Int Endod J. 2005; 38(1):2-7.

25. Ezzie E, Fleury A, Solomon E, Spears R, He J. Efficacy of retreatment techniques for a resin based root canal obturation material. J Endod. 2006; 32(4):341-4.

26. Mollo A, Botti G, Principi GN, Randellini E, Paragliola R, Chazine M, Ounsi HF, Grandini S. Efficacy of two Ni-Ti systems and hand files for removing gutta-percha from root canals. Int Endod J. 2012; 45(1):1-6.

27. Ferreira JJ, Rhodes JS, Ford TR. The efficacy of gutta-percha removal using ProFiles. Int Endod J. 2001; 34(4):267-74.

28. Imura N, Kato AS, Hata GI, Uemura M, Toda $\mathrm{T}$, Weine $\mathrm{F}$. A comparison of the relative efficacies of four hand and rotary instrumentation techniques during endodontic retreatment. Int Endod J. 2000; 33(4):361-66.

29. Barletta FB, Rahde Nde M, Limongi O, Moura $\mathrm{AA}$, Zanesco $\mathrm{C}$, Mazocatto G. In vitro comparative analysis of 2 mechanical techniques for removing gutta-percha during retreatment. J Can Dent Assoc. 2007; 73(1):65.

30. Rodig T, Hausdorfer T, Konietschke F, Dullin C, Hahn W, Hulsmann M. Efficacy of D-RaCe and ProTaper Universal Retreatment NiTi instruments and hand files in removing guttapercha from curved root canals- a microcomputed tomography study. Int Endod J. 2012; 45(6):580-89.

31. Zuolo AS, Mello JE Jr, Cunha RS, Zuolo ML, Bueno CE. Efficacy of reciprocating and rotary techniques for removing filling material during root canal retreatment. Int Endod J. 2013; 46(10):947-53.

32. Vidal FT, Nunes E, Horta MC, Freitas MR, Silveira FF. Evaluation of three different rotary systems during endodontic retreatment - 
Analysis by scanning electron microscopy. J Clin Exp Dent. 2016; 8(2):125-29.

33. Kim S, Baek S. The microscope and endodontics. Dent Clin North Am. 2004; 48(1):11-8.

34. Schirrmeister JF, Meyer KM, Hermanns P, Altenburger MJ, Wrbas KT. Effectiveness of hand and rotary instrumentation for removing a new synthetic polymer-based root canal obturation material (Epiphany) during retreatment. Int Endod J. 2006; 39(2):150-56.

35. Marques da Silva B, Baratto-Filho F, Leonardi DP, Henrique Borges A, Volpato L, Branco Barletta F. Effectiveness of ProTaper, D-RaCe, and Mtwo retreatment files with and without supplementary instruments in the removal of root canal filling material. Int Endod J. 2012; 45(10):927-32.

36. Alves FR, Almeida BM, Neves MA, Moreno JO, Rôças IN, Siqueira-Junior JF. Disinfecting oval-shaped root canals: effectiveness of different supplementary approaches. J Endod. 2011; 37(4):496-501.

\section{CONFLITO DE INTERESSES}

Os autores declaram não haver conflitos de interesse.

\section{AUTOR PARA CORRESPONDENCIA}

\section{Marcos Sérgio Endo}

marcossendo@gmail.com

Submetido em 29/05/2018

Aceito em 27/06/2018 